\title{
Von der Revolution \\ zur Partizipation: Social Media und der demokratische Willensbildungsprozess
}

\author{
DOI 10.1007/s11576-011-0279-X
}

\section{Der Autor}

Prof. Dr. Hans Ulrich Buhl ( $\varangle)$

FIM Kernkompetenzzentrum Finanz-

\& Informationsmanagement

Universität Augsburg

Universitätsstraße 12

86159 Augsburg

Deutschland

mailto:hans-ulrich.buhl@wiwi.uni-

augsburg.de

Online publiziert: 2011-06-16

This article is also available in English via http://www.springerlink.com and http://www.bise-journal.org: Buhl HU (2011) From Revolution to Participation: Social Media and the Democratic Decision-Making Process. Bus Inf Syst Eng. doi: 10.1007/s12599-011-0166-4.

(C) Gabler Verlag 2011
„Ägypten ist offline“. Als diese Nachricht am 28.01.2011 über die Ticker lief, hatte die sogenannte „Facebook-Revolution“ einen neuen Höhepunkt erreicht. Nach Ansicht des zu diesem Zeitpunkt noch herrschenden Präsidenten Husni Mubarak und seines Regimes war es nicht mehr ausreichend, vereinzelt Mobilfunknetze und Social-MediaDienste wie Facebook, Twitter und Co. zu blockieren. Vielmehr schien das gesamte Internet zu einer nicht mehr zu kontrollierenden Gefahr für den staatlichen Machtapparat geworden zu sein, die diesen drastischen und bisher einmaligen Schritt notwendig machte (Kremp 2011). Was war geschehen?

Das Phänomen der Verbreitung von Informationen gegen den Willen autoritärer Regime mittels Social-Media-Diensten war nicht neu. Im Anschluss an die Präsidentschaftswahl im Iran gelangten beispielsweise bereits im Jahr 2009 Nachrichten über die Proteste der Opposition fast ausschließlich über die Microblogging-Plattform Twitter und das Videoportal YouTube an die ausländische Öffentlichkeit (Meller 2011). Während in Tunesien schon seit Ende des Jahres 2007 das Videoportal YouTube nicht mehr erreichbar war, begann sich im Jahr 2010 der Unmut der Bevölkerung im sozialen Netzwerk Facebook zu kanalisieren (Lobo 2011). Dabei wurden zu Beginn hauptsächlich Informationen über die Missstände im durch das korrupte Regime von Zine el-Abidine Ben Ali heruntergewirtschafteten Land ausgetauscht (z. B. über das soziale Ungleichgewicht, die immense Zahl an (insbesondere jungen) Arbeitslosen, die weit verbreitete Willkür der Beamten etc.). Im Laufe der Zeit wurden zunehmend auch gezielt Protestveranstaltungen über Social-Media-Dienste organisiert und Nachrichten über den Verlauf der Proteste verbreitet. So wurde Facebook die zentrale Kommunikationsplattform der Proteste, wobei sich innerhalb von zwei Monaten über 300.000 Tunesier neu registrierten (Lobo 2011). Dies stellt bei gerade einmal vier Millionen Menschen mit Internetzugang durchaus eine enorme Zahl dar. Während das Regime durch den Diebstahl von Facebook-Passwörtern und gezielte Desinformation noch versuchte, den Lauf der Dinge zu beeinflussen (Madrigal 2011), sah sich Tunesiens Präsident Zine el-Abidine Ben Ali am 14. Januar 2011 endgültig zur Flucht gezwungen. Obwohl Social-MediaDiensten wie Facebook vor allem die Rolle eines Katalysators für den sich über Jahre im Untergrund aufgestauten öffentlichen Unmut zugeschrieben wird (Lobo 2011), lenkten erst diese die geballte Aufmerksamkeit traditioneller Medien wie Rundfunk und Fernsehen auf die Geschehnisse. Die flächendeckende Verbreitung der Nachrichten über die Aufstände in der arabischen Welt wurde im Anschluss vor allem durch den arabischen Nachrichtensender Al-Jazeera vorangetrieben (Meller 2011). Wenige Wochen später, am 11. Februar 2011, trat in der Folge auch Ägyptens Präsident Husni Mubarak zurück. Mit ca. 5,7 Millionen registrierten Facebook-Nutzern (o.V. 2011c) und einer Vielzahl an weiteren intensiv genutzten Social-Media-Diensten wie Twitter oder dem Windows Live Messenger (vormals MSN) spielte auch dort die digitale Vernetzung der Menschen eine entscheidende Rolle (Meller 2011).

Aus Angst, dass sich die Vorfälle in Nordafrika und im Nahen Osten wiederholen könnten, reagieren vielerorts Regierungen mit präventiven Gegenmaßnahmen oder versuchen, Social-Media-Dienste für ihre Zwecke zu instrumentalisieren. Nachdem beispielsweise in China bereits seit einigen Jahren zahlreiche Social-Media-Dienste nur noch eingeschränkt oder gar nicht mehr verfügbar sind, wurde kurz nach Ausbruch der Protestwellen aus Angst vor dem Suchbegriff „Jasminrevolution“ u. a. auch das auf geschäftliche Kontakte abzielende soziale Netzwerk LinkedIn gesperrt (o.V. 2011a). Gleichzeitig setzen einige Staaten darauf, selbst solche Dienste aufzubauen, da sich diese in der Regel leichter kontrollieren und zensieren lassen (Morozov 2011, S. 13). Beispiele hierfür lassen sich $u$. a. in Russland und einigen ehemaligen GUS Staaten (z. B. http://vkontakte.ru) sowie in Vietnam (z. B. http://go.vn) finden. In Bahrain versuchte die Regierung hingegen, bestehende Social-Media-Dienste zu manipulieren, in dem 
beispielsweise kurz nach den ersten Protesten im eigenen Land Twitter mit regierungsfreundlichen Nachrichten überflutet wurde, um so die Glaubwürdigkeit des Nachrichtendienstes zu untergraben (Morozov 2011, S. 13). Diese Maßnahmen verdeutlichen den immensen Respekt insbesondere autoritärer Regime vor einer Machtverschiebung in der Gesellschaft weg von einzelnen Informationsanbietern hin zu einem engmaschigen und dynamischen Netzwerk aus beliebig vielen Anbietern und Nachfragern. Diese Entwicklung ermöglicht es, auch etablierte Machtinstanzen in Frage zu stellen (Meller 2011). So scheinen Social-Media-Dienste den demokratischen Willensbildungsprozess in autoritären Staaten zu unterstützen und zu beschleunigen.

Auch in etablierten Demokratien versuchen Politiker, auf die gestiegene Bedeutung von Social-Media-Diensten zu reagieren und diese positiv für sich zu nutzen. So werden u. a. Wahlkämpfe nicht mehr nur über das Fernsehen, Plakate und Wahlkampfveranstaltungen ausgetragen, sondern auch im Internet. In einer Untersuchung des privaten Forschungsinstituts Pew Internet \& American Life Project gaben im Jahr 2008 ca. 46 \% aller befragten US-Bürger an, mittels Internet, E-Mail oder Mobilfunk Wahlnachrichten erhalten, ihre Ansichten mit anderen ausgetauscht oder andere Wähler mobilisiert zu haben (Smith und Lee 2008, S. v). Darüber hinaus sahen sich ca. 35 \% der Befragten Online-Videos im Zusammenhang mit den Wahlen an und ca. $10 \%$ nutzen soziale Netzwerke im Web für ihr politisches Engagement (Smith und Lee 2008, S. v). Dabei galt Barack Obama als der perfekte Internet-Kandidat (Klippstätter 2008), der es als einer der Ersten verstand, das Potenzial von Social-Media-Diensten für sich zu nutzen. Während beispielsweise die digitale Pinnwand auf Obamas Facebook-Seite zu Wahlkampfzeiten bereits mehr als 14.000 Einträge verzeichnete (Schnoor 2008), konnte er im Juli 2010 bereits mehr als 10 Mio. digitale Fans für sich gewinnen (o.V. 2010a). Auch in Deutschland werden Social-Media-Dienste zunehmend für den sogenannten Wahlkampf 2.0 genutzt, wobei laut einer Untersuchung des Hightech-Verbands BITKOM im Wahljahr 2009 alle Spitzenpolitiker und Parteien vertreten waren (o.V. 2009). Dabei konnte die deutsche Bundeskanzlerin Angela Merkel mit ca. 80.000 Unterstützern die größte Anzahl an „virtuellen“ Anhängern aufweisen (o.V. 2009). Mittlerweile wurde sie von ihrem Ex-Bundesverteidigungsminister Karl-Theodor zu Guttenberg abgelöst. Nachdem diesem in weiten Teilen seiner Dissertation der Vorwurf des Plagiarismus nachgewiesen werden konnte, hatte sich zu Guttenberg nach seinem Rücktritt mittels Video-Botschaft an seine Unterstützer im Internet gewandt. Diese umfassen allein auf der Facebook-Seite „Wir wollen Guttenberg zurück“ beinahe 590.000 Anhänger. Der Fall Guttenberg zeigt jedoch auch die Kehrseite der Medaille. Ohne den „Aufstand im Netz" (o.V. 2011b), der mittels Social-Media-Diensten erst richtig ins Rollen kam, hätte sich der bis dahin ungemein beliebte Politiker trotz seines wissenschaftlich inakzeptablen Verhaltens gegebenenfalls im Amt halten können. So kam erst durch die detaillierte Recherche einer Vielzahl an u. a. „Hobby-Plagiatsjägern“ auf der SocialMedia-Plattform Guttenplag Wiki das gesamte Ausmaß der Affäre ans Licht und in das Bewusstsein der breiten Bevölkerung (o.V. 2011b). Auch ein offener Brief und eine Unterschriftenliste von (Nachwuchs-) Wissenschaftlern wurde auf diese Weise organisiert und publiziert, wodurch sich der öffentliche Druck weiter erhöhte (o.V. 2011b), der letztendlich einen Rücktritt als unausweichlich erscheinen ließ. Aber auch personenunabhängige Themen wie das umstrittene Projekt „Stuttgart $21^{\text {“ }}$ - der Bau eines neuen unterirdischen Bahnhofs in der Landeshauptstadt Stuttgart - stehen bei den Nutzern von Social-Media-Diensten weit oben auf der Agenda. So sprachen sich in der mit rund 1,2 Mio. Teilnehmern deutschlandweit größten jemals durchgeführten Umfrage in den sozialen Netzwerken SchülerVZ, studiVZ und meinVZ rund 650.000 Menschen gegen das Bauvorhaben aus (o.V. 2010c). Während Social-Media-Dienste bisher hauptsächlich die Zielgruppe der 18 bis 29 jährigen Wähler adressierten, zeichnet sich ein Trend hin zu einem steigenden Altersdurchschnitt ab. Beispielsweise sind laut einer aktuellen Untersuchung ca. $58 \%$ aller Nutzer in 19 untersuchten sozialen Netzwerken im Web 35 Jahre oder älter (o.V. 2010b). Diese Entwicklung dürfte zukünftig den Einfluss von Social-Media-Diensten auf den politischen Willensbildungsprozess auch in etablierten Demokratien weiter steigern und eine erhöhte Aufmerksamkeit erfordern, um den unterschiedlichen Informationsbedürfnissen der jeweiligen Altersgruppen gerecht zu werden.

Einige westliche Demokratien sind hier bereits einen Schritt weiter und ermöglichen der Bevölkerung bereits frühzeitig eine Partizipation an Entscheidungsprozessen mittels Social-Media-Diensten. Beispielsweise wurde in den letzten Jahren im Zuge der deutschen Bundesinitiative E-Government 2.0 u. a. die Umsetzung von 
E-Partizipationsprojekten gefördert. Ein konkretes Beispiel für eine erfolgreiche Implementierung ist die Plattform e-konsultation.de (http://e-konsultation.de). Hier werden seit Februar 2008 Online-Konsultationen durchgeführt, um der deutschen Bevölkerung die Möglichkeit zu geben, sich aktiv an der Gestaltung von politischen Themen und Vorhaben zu beteiligen. Weitere Beispiele finden sich in Deutschland in den zuletzt implementierten Bürgerdialogen „Nachhaltigkeit“ (http://www.dialog-nachhaltigkeit.de), „Aufwachsen mit dem Netz“ (http://www.dialog-internet.de) und „Aufbruch Bayern“ (http://www.archiv.aufbruch-bayern.de/start.php). Im Rahmen des letztgenannten Beispiels wurde ein achtwöchiger öffentlicher Online-Dialog zwischen den Bürgerinnen und Bürgern des Freistaats Bayerns und der Bayerischen Staatsregierung angeboten. Mit Hilfe verschiedener Social-Media-Dienste wie Facebook, Twitter und YouTube diskutierten und bewerteten 100.000 Teilnehmer 740 nutzergenerierte Vorschläge und Ideen zu den Rubriken „Familie“, „Bildung“ und „Innovation“. Die besten Beiträge wurden schließlich im Bayerischen Ministerrat hinsichtlich ihrer Umsetzbarkeit diskutiert. Aber auch international setzen immer mehr Regierungen auf die Partizipation der Bürger mittels Social-Media-Diensten. So lassen sich nicht nur in UK mit „Citizen Space“ (http://www.citizenspace.com) und „E-Community Council“ (http://www.ecommunitycouncil.org.uk) Beispiele zur E-Partizipation finden. Auch in Australien werden die Bürger mittels Social-Media-Diensten zur Beteiligung an OnlineKonsultationen, beispielsweise bezüglich der zukünftigen Gestaltung von Parks in Victoria (http://www.weplan.parks.vic.gov.au), ermutigt. Im Rahmen dieser Art der Bürgerbeteiligung werden neben regionalen Themen einzelner Städte oder Gemeinden oftmals auch nationale Belange, beispielsweise bezüglich der Gesetzgebung, oder sogar länderübergreifende Themen, beispielsweise im Zusammenhang mit Krisenregionen, diskutiert und oft in darauf folgenden Entscheidungsprozessen berücksichtigt. Stellen Social-Media-Dienste somit die allheilbringende Wunderwaffe im demokratischen Willensbildungsprozess dar?

Grundsätzlich gilt es, den vermehrten Einsatz von Social-Media-Diensten im politischen wie auch im gesellschaftlichen Leben als Informationsquelle der Zukunft kritisch zu hinterfragen. Die oftmals eingeschränkte bzw. aus dem Kontext gelöste Sicht auf die Wirklichkeit birgt große Potenziale für Miss- und Fehlinterpretationen bzw. für einseitige und stark verzerrte Wahrnehmungen der Realität. So werden die in Social-MediaDiensten bereitgestellten Informationen einerseits durch die implementierten Algorithmen zur Informationsaufbereitung der Dienste selbst (wie z. B. im Fall Facebook) und andererseits durch die selbstständige Selektion der Informationsquellen bzw. Themenfelder (wie z. B. im Fall Twitter) gefiltert bzw. eingeschränkt (Lischka 2011). Daneben werden oft auch abweichende oder nicht als konform erscheinende Meinungen gar nicht oder nur in seltenen Fällen über Social-Media-Dienste kommuniziert (Lischka 2011) bzw. der freie Zugang zu diesen eingeschränkt. Die dadurch oftmals bedingte einseitige Sichtweise kann subjektive Wahrnehmungen und Eindrücke der Nutzer überproportional verstärken. Zudem werden Informationen in einigen Social-MediaDiensten wie Twitter oftmals nur in Form sogenannter Microblogs mit einer maximalen Länge von 140 Zeichen bereitgestellt. Dies führt dazu, dass die übermittelten Informationen i. d. R. nicht in einen breiteren Kontext eingebettet sind und somit großen Spielraum für unterschiedlichste Interpretationen bieten. Was ist also zu tun, um die Potenziale von Social-Media-Diensten im demokratischen Willensbildungsprozess zu nutzen sowie Risiken rechtzeitig zu erkennen und zu minimieren bzw. zu vermeiden?

Aus dieser Frage lassen sich zahlreiche Herausforderungen sowohl für die Politik als auch die Wirtschaftsinformatik (WI) als Disziplin ableiten:

- Im Zuge der immer stärkeren Verbreitung von Social-Media-Diensten über alle Alters- und Gesellschaftsgruppen hinweg muss sich die Politik zunehmend mit dem Thema Social Media im Rahmen des demokratischen Willensbildungsprozesses auseinandersetzten. Während in autoritären Staaten ein freier Zugang von SocialMedia-Diensten im Hinblick auf die Möglichkeit zur demokratischen Partizipation höchste Priorität haben sollte, stehen auch etablierte Demokratien und deren Politiker vor Herausforderungen. Besonders auf persönlicher Ebene gilt es, die eigene Authentizität zu wahren und die eigene Persönlichkeit nicht auf dem Altar einer falsch verstandenen Selbstinszenierung und um des Populismus Willen zu opfern. Im Rahmen des E-Government ist hingegen darauf zu achten, Möglichkeiten zur Partizipation an Entscheidungsprozessen wie E-Partizipation zielgerichtet einzusetzen, diese komplementär zu etablierten Expertengremien zu sehen und diese nicht als allheilbringendes Substitut zu überschätzen. So kann E-Partizipation sicherlich helfen, 
wertvolle Ideen und Anregungen von Seiten der Bürger zu sammeln und Entscheidungsprozesse damit nachvollziehbarer zu gestalten. Allerdings muss E-Partizipation dazu beispielsweise derart ausgestaltet sein, dass sich nicht nur Experten, sondern auch „normale“ Internetnutzer darin wiederfinden (de Maizière 2010), um einen zielführenden Beitrag leisten zu können. Andererseits wird es immer Entscheidungen geben, die aus lokaler Sicht unpopulär, mit Blick auf das große Ganze jedoch unvermeidbar sind. Auch an dieser Stelle werden auf das Konzept der E-Partizipation und auf Grund der rasanten Verbreitung von Social-Media-Diensten auf die Politik immense Herausforderungen zukommen.

- Im Hinblick auf den Einsatz von Social-Media-Diensten im Rahmen des demokratischen Willensbildungsprozesses kann die WI auf Grund ihres Methodenpluralismus und ihrer ausgeprägten Interdisziplinarität besonders wertvolle Beiträge leisten. So gilt es beispielsweise mit Hilfe finanzwirtschaftlicher Methoden, den Einsatz und die Gestaltung von Social-Media-Diensten zur E-Partizipation zu bewerten, damit Nutzen und Kosten bzw. Chancen und Risiken in einem ökonomisch sinnvollen Verhältnis zueinander stehen. Des Weiteren sind zahlreiche Fragestellungen bezüglich des freien Zugangs zu Social-Media-Diensten sowie der Sicherheit und Glaubwürdigkeit von zur Verfügung gestellten Daten und Informationen kritisch zu diskutieren. Auch an dieser Stelle kann die WI durch quantitative und qualitative Forschungsarbeiten sowie durch die enge Zusammenarbeit verschiedener Disziplinen wie Informatik, Rechtswissenschaften etc. zu einem theoretischen Erkenntnisfortschritt, aber insbesondere auch zu praxisrelevanten Artefakten beitragen. Zudem gilt es, geeignete Algorithmen für die sinnvolle Filterung und Bündelung von Informationen zu entwickeln, um zum einen die einseitige Verbreitung von Informationen und den damit einhergehenden Informationsverlust abzuwenden und zum anderen eine bessere Einbettung in den Gesamtkontext zu gewährleisten. Aber auch Fragen der sowohl inhaltlichen als auch technischen Integration von i. d. R. durch Dritte betriebenen Social-Media-Diensten in bestehende Informations- und Kommunikationssysteme und der Nutzerakzeptanz bergen aus wissenschaftlicher wie praktischer Sicht hohes Potenzial für zukünftige Forschungsarbeiten. Auch hier bietet das breite Methodenspektrum der WI mit ihren vielfältigen technoökonomischen Forschungsmethoden eine sehr gute Ausgangsbasis.

\section{Literatur}

de Maizière $O$ (2010) Online-Konsultationen sind kein demokratischer Selbstläufer. http:// www.spiegel.de/netzwelt/netzpolitik/0,1518,731118,00.html. Abruf am 2011-03-14

Klippstätter K (2008) Obama ist der perfekte Internet-Kandidat. http://www.computerwoche.de/ netzwerke/web/1875977/index3.html. Abruf am 2011-03-14

Kremp M (2011) Wie Ägypten aus dem Internet verschwand. http://www.spiegel.de/netzwelt/ netzpolitik/0,1518,742232,00.html. Abruf am 2011-03-14

Lischka K (2011) Die ganze Welt ist meiner Meinung. http://www.spiegel.de/netzwelt/web/0, 1518,750111,00.html. Abruf am 2011-04-06

Lobo S (2011) Wie Facebook arabische Online-Spitzel besiegte. http://www.spiegel.de/ netzwelt/web/0,1518,742961,00.html. Abruf am 2011-03-14

Madrigal A (2011) The inside story of how Facebook responded to Tunisian hacks. http://www. theatlantic.com/technology/archive/2011/01/the-inside-story-of-how-facebook-respondedto-tunisian-hacks/70044/. Abruf am 2011-03-14

Meller P (2011) Twitter, Facebook und die Revolution in Ägypten. http://www.cicero.de/97. php?ress_id=1\&item $=5848$. Abruf am 2011-03-14

Morozov E (2011) Kluge Diktatoren. Süddeutsche Zeitung 2011(59):13

oV (2009) Angela Merkel liegt im Wahlkampf 2.0 vorne. http://www.bitkom.org/de/themen/ 57545_60824.aspx. Abruf am 2011-03-14

oV (2010a) Obama hat fast so viele Fans wie Lady Gaga. http://www.spiegel.de/panorama/ gesellschaft/0,1518,705089,00.html. Abruf am 2011-03-14

oV (2010b) Study: ages of social network users. http://royal.pingdom.com/2010/02/16/ study-ages-of-social-network-users/. Abruf am 2011-03-15

oV (2010c) VZ-Netzwerke: Deutschlands größte Umfrage zu Stuttgart 21 mit 1,2 Millionen Teilnehmern/Über 52 Prozent stimmen gegen das umstrittene Projekt. http://www. presseanzeiger.de/meldungen/gesellschaft-kultur/401247.php. Abruf am 2011-03-14

oV (2011a) Angst vor Revolutionsgeist - China blockiert auch Business-Netzwerk LinkedIn. http://www.spiegel.de/netzwelt/netzpolitik/0, 1518,747705,00.html. Abruf am 2011-03-14

oV (2011b) Guttenberg dankt seinen Facebook-Freunden. http://www.abendblatt.de/politik/ article1827654/Guttenberg-dankt-seinen-Facebook-Freunden.html. Abruf am 2011-03-14 oV (2011c) http://www.checkfacebook.com/. Abruf am 2011-03-14

Schnoor F (2008) Obama und seine Freunde. http://zuender.zeit.de/2007/34/us_praesident schaftswahl_barack_obama_online. Abruf am 2011-03-14

Smith A, Lee R (2008) The Internet and the 2008 election. Pew Internet \& Amarican Life Project, Washington, DC 